\title{
A probabilistic representation for the solutions to some non-linear PDEs using pruned branching trees
}

\author{
D. Blömker ${ }^{\mathrm{a}}, \mathrm{M}$. Romito ${ }^{\mathrm{b}, *}, \mathrm{R}$. Tribe ${ }^{\mathrm{c}}$ \\ a Institut für Mathematik, RWTH Aachen, Templergraben 55, 52052 Aachen, Germany \\ b Dipartimento di Matematica “U. Dini”, viale Morgagni 67/a, 50134 Firenze, Italia \\ ${ }^{\mathrm{c}}$ University of Warwick, Mathematics Institute, CV4 7AL Coventry, UK
}

Received 26 May 2005; received in revised form 25 November 2005; accepted 7 February 2006

Available online 25 September 2006

\begin{abstract}
The solutions to a large class of non-linear parabolic PDEs are given in terms of expectations of suitable functionals of a tree of branching particles. A sufficient, and in some cases necessary, condition is given for the integrability of the stochastic representation, using a comparison scalar PDE.

In cases where the representation fails to be integrable, a sequence of pruned trees is constructed, producing approximate stochastic representations that in some cases converge, globally in time, to the solution of the original PDE.
\end{abstract}

(c) 2006 Elsevier Masson SAS. All rights reserved.

\section{Résumé}

Les solutions d'une latge classe EDP non linéaires paraboliques sont données sous la forme d'espérances de fonctionnelles adéquatement choisies d'un arbre de branchement. L'intégrabilité est établie par comparaison avec une EDP scalaire. Lorsque l'intégrabilité fait défaut, un procédé d'élagage permet dans certains cas de produire une suite convergeant vers la solution de l'EDP d'origine.

(c) 2006 Elsevier Masson SAS. All rights reserved.

MSC: primary 60J80; secondary 35Q30, 35K55, 35C $99,76 \mathrm{M} 35,60 \mathrm{H} 30$

Keywords: Branching processes; Pruned trees; Stochastic representation; Burgers equation; Navier-Stokes equations; Semi-implicit approximation

\section{Introduction}

This paper considers stochastic representations for solutions to a large class of non-linear parabolic PDEs, or systems of PDEs, of the type

$$
\partial_{t} u=A u+\mathcal{F}(u)+f,
$$

\footnotetext{
* Corresponding author.

E-mail addresses: bloemker@instmath.rwth-aachen.de (D. Blömker), romito@math.unifi.it (M. Romito), tribe@maths.warwick.ac.uk
} (R. Tribe). 
where $A$ is a linear operator with a complete set of eigenfunctions, $\mathcal{F}$ is a polynomial nonlinearity in $u$ and its derivatives, and $f$ is a given driving function. In short, the solution $u$ is expanded into a Fourier series using the eigenfunctions of $A$. This yields (as in spectral Galerkin methods) a system of countably many coupled ODEs for the Fourier coefficients. This ODE system is then solved in a weighted $\ell^{\infty}$-space, via an expectation over a tree of branching particles. The rules for the branching and dying probabilities arise from the particular PDE being studied. Moreover the PDE determines an evaluation operator $R_{t}$, acting on the tree $\mathcal{T}_{\mathbf{k}}$ of particles rooted at each Fourier mode $\mathbf{k}$, so that, under integrability assumptions, the (suitably weighted) $\mathbf{k t h}$ Fourier mode $\chi_{\mathbf{k}}(t)$ is given by

$$
\chi_{\mathbf{k}}(t)=\mathrm{E}\left[R_{t}\left(\mathcal{T}_{\mathbf{k}}\right)\right]
$$

This is precisely the method of Le Jan and Sznitman [10,11] where they treated the Navier-Stokes equations in $\mathbf{R}^{3}$. We comment on related literature later in this introduction.

\subsection{Integrability}

The major drawback of the stochastic representation is that it often fails to exist for large times $t$, although the solution to the PDE may still exist. The problem is that $R_{t}\left(\mathcal{T}_{\mathbf{k}}\right)$ may fail to be an integrable random variable for $t \geqslant t_{0}$. The standard way to check integrability is to establish a scalar real-valued comparison equation. The finiteness of this comparison equation implies the integrability needed for the stochastic representation to hold (and in some cases provides a sufficient condition as well). However, the comparison equation represents a worst case scenario with super-linear (explosive) growth and it ignores most of the structure of the non-linearity in the original PDE. It typically leads to a representation that holds for all $t \geqslant 0$ only for small initial data.

Our main aim is to present an approach to treat cases where integrability fails. Our approach is to construct sets $\Omega_{n}$, with $\mathrm{P}\left[\Omega_{n}\right] \uparrow 1$ so that

$$
\chi_{\mathbf{k}}(t)=\lim _{n \rightarrow \infty} \mathrm{E}\left[R_{t}\left(\mathcal{T}_{\mathbf{k}}\right) \mathbf{1}_{\Omega_{n}}\right] .
$$

This treats the expectation somewhat as a singular integral, where we have to be careful how to cut out the singularity. The method we use, explained in Section 5, is to construct a pruned branching tree $\mathcal{T}_{\mathbf{k}}^{(n)}$ which will agree with $\mathcal{T}_{\mathbf{k}}$ on $\Omega_{n}$. The expectation for the pruned tree

$$
\mathrm{E}\left[R_{t}\left(\mathcal{T}_{\mathbf{k}}^{(n)}\right)\right]=\mathrm{E}\left[R_{t}\left(\mathcal{T}_{\mathbf{k}}\right) \mathbf{1}_{\Omega_{n}}\right]
$$

is always well defined and will represent the kth Fourier mode of the solution to a semi-implicit approximation scheme of the type

$$
\partial_{t} u^{(n)}=A u^{(n)}+\widetilde{\mathcal{F}}\left(u^{(n)}, u^{(n-1)}\right)+f .
$$

We then use PDE techniques to verify that the approximation scheme converges to a solution of the original PDE. Although there are general results for the convergence of such approximations (see for example Bjørhus and Stuart [5]) the assumptions are usually quite restrictive. Since stronger arguments are specific to the particular PDE being studied, we present the arguments only in one representative case, namely for Burgers equation. In this example the limiting probabilistic representation (1.2) holds for all times $t \geqslant 0$. Currently, only in a very simple framework of small initial conditions and uniformly small forcing is it currently possible to derive such representation results for large times (for the 3D Navier-Stokes equations see Bakhtin [3] and Waymire [21]).

\subsection{A toy example}

We illustrate here the key idea of the pruning scheme on the simplest example possible, namely the ordinary differential equation $\dot{u}=-u+u^{2}$. The solution can be given by the stochastic representation $u(t)=\mathrm{E}\left[u(0)^{N_{t}}\right]$, where $N_{t}$ is the number of particles at time $t$ of a simple rate one binary branching process, starting from a single particle at time 0 . It is easy to verify that the representation is well defined for all times $t \geqslant 0$ if and only if $|u(0)| \leqslant 1$, in that the variable $|u(0)|^{N_{t}}$ becomes non-integrable for large $t$ when $|u(0)|>1$, while the solutions of the equation blow up only if $u(0)>1$.

We now give a modification of the branching process. Give each particle a label from the integers N. Particles still branch at rate 1 but a particle with label $n$ produces two offspring, one with label $n$ and one with label $n-1$. When 
a particle of type 0 tries to branch it simply dies. Start with a single particle with label $n$ and let $N_{t}(n)$ denote the total number of particles at time $t$, regardless of their label. Set $u_{n}(t)=\mathrm{E}\left[u(0)^{N_{t}(n)}\right]$ for $n \geqslant 0$ and $u_{-1} \equiv 0$. Then it is possible to show that $u_{n}(t)$ solves the following semi-implicit iterative scheme

$$
\dot{u}_{n}=-u_{n}+u_{n-1} u_{n}, \quad u_{n}(0)=u(0), \quad \text { for } n \geqslant 0 .
$$

It is straightforward to check that $u_{n}(t)$ is well defined for all $n$ and $t$. Moreover, $u_{n}$ converges to the solution $u(t)$ of the original problem for each initial condition $u(0) \leqslant 1$. This yields the stochastic representation

$$
u(t)=\lim _{n \rightarrow \infty} \mathrm{E}\left[u(0)^{N_{t}(n)}\right]
$$

valid for all $u(0) \leqslant 1$ and all $t \geqslant 0$.

Remark 1.1. The seemingly simpler modification (used by Le Jan and Sznitman [10] for their uniqueness proof and by Bhattacharya et al. [4]) where a particle with label $n$ produces two offspring each with label $n-1$, leads to the explicit iterative scheme $\dot{u}_{n}=-u_{n}+u_{n-1}^{2}$. Unfortunately, the limit of $u_{n}(t)$ for large $t$, as $n \rightarrow \infty$, fails to exist for $u(0)<-1$.

The semi-implicit approximation scheme works for other polynomial non-linearities. For example, if one considers $\dot{u}=-u-u^{3}$, the approximation scheme $u_{n}=-u_{n}-u_{n-1}^{2} u_{n}$, where each particle with label $n$ branches into three particles, one with label $n$ and two with label $n-1$, is convergent to the true global solution for any initial condition.

\subsection{Related literature}

Earlier papers connecting branching particle systems to PDEs (for instance Skorokhod [18] or Ikeda, Nagasawa and Watanabe [9], and later McKean [13]) use branching coupled with a diffusion, and the stochastic representation is derived directly without Fourier series, so that the linear operator $A$ is limited to generators of Markov processes. The idea of representing the Fourier modes, from Le Jan and Sznitman [10], was later extended in Bhattacharya et al. [4], Chen et al. [6], Waymire [21], and to a representation in the physical space in Ossiander [16].

When deriving a system of ODEs in $\ell^{\infty}$ space, there is considerable freedom in the choice of weights for the Fourier coefficients. See Bhattacharya et al. [4] for an extensive discussion in the case of 3D Navier-Stokes. We establish a comparison equation for our examples in Section 4. We show that for a class of equations, which includes Burgers but not 3D Navier-Stokes, that the choice of weights cannot affect the integrability of the probabilistic representation.

Pruning of the random trees also appears in the literature. In Bhattacharya et al. [4] the branching trees are pruned after $n$ generations. This gives a stochastic representation of a Picard iteration scheme converging to the original PDE, but, as stated in [4], the existence of the expectation is equivalent to the convergence of the Picard iteration scheme. In another approach to non-integrability, Morandin [15] suggested a clever re-summation of the expectation in order to improve the convergence for large times, but he was only able to rigorously verify the global convergence of his method in a simple example where (1.1) is a one-dimensional ODE.

\subsection{Layout of the paper}

The paper is organised as follows. In Section 2 we present our abstract ODE setting and show how to recast three representative examples of PDEs with quadratic non-linearities into this required form. In Section 3 we develop the notation needed for the random trees and establish the stochastic representation under the assumption that it is integrable. In Section 4 we investigate the comparison equation which typically shows the representation is integrable at small times, or, when there is no linear instability, for all times with small data. For a class of equations we obtain necessary and sufficient conditions for the integrability of the stochastic representation that are independent of the choice of weights in Fourier space. In Section 5 we develop the idea of pruning and apply it to the case of the 1D Burgers equation.

\section{Abstract setting and examples}

We first present an infinite system of ODEs involving a quadratic non-linearity. The system is indexed over $\mathbf{k} \in \mathbf{Z}^{d}$. We then discuss several examples of PDEs on the torus $[0,2 \pi)^{d}$ and recast their Fourier transforms into our abstract 
ODE setting. We do not present the highest generality possible, but focus instead only on an equation with one quadratic nonlinearity, one additional linear part and one forcing term.

\subsection{The general system of ODEs}

We consider solutions $\chi(t): \mathbf{Z}^{d} \rightarrow \mathbf{C}^{r}$ to the following infinite dimensional system of $\mathbf{C}^{r}$-valued ODEs

$$
\dot{\chi}_{\mathbf{k}}=\lambda_{\mathbf{k}}\left[-\chi_{\mathbf{k}}+C_{R} p_{\mathbf{k}} \chi_{\mathbf{k}}+C_{B} \sum_{\mathbf{l}, \mathbf{m} \in \mathbf{Z}^{d}} q_{\mathbf{k}, \mathbf{l}, \mathbf{m}} B_{\mathbf{k}, \mathbf{l}, \mathbf{m}}\left(\chi_{\mathbf{l}}, \chi_{\mathbf{m}}\right)+d_{\mathbf{k}} \gamma_{\mathbf{k}}\right]
$$

with $\mathbf{k} \in \mathbf{Z}^{d}$. The constants $\lambda_{\mathbf{k}}>0$ (which will determine the rate of particle evolution), $p_{\mathbf{k}}, q_{\mathbf{k}, \mathbf{l}, \mathbf{m}}, d_{\mathbf{k}} \in[0,1]$ (which will determine the probabilities of regeneration, branching and dying), and $C_{R}, C_{B} \geqslant 0$ (the regeneration and branching constants) are fixed, as are bilinear operators $B_{\mathbf{k}, \mathbf{l}, \mathbf{m}}: \mathbf{C}^{r} \times \mathbf{C}^{r} \rightarrow \mathbf{C}^{r}$ satisfying

$$
\left|B_{\mathbf{k}, \mathbf{l}, \mathbf{m}}\left(\chi, \chi^{\prime}\right)\right| \leqslant|\chi|\left|\chi^{\prime}\right|
$$

for all $\chi, \chi^{\prime} \in \mathbf{C}^{r}$. The choice of these constants will arise from the Fourier transform of the PDE being studied. We assume throughout that

$$
p_{\mathbf{k}}+q_{\mathbf{k}}+d_{\mathbf{k}}=1 \quad \text { for all } \mathbf{k} \in \mathbf{Z}^{d},
$$

and

$$
p_{\mathbf{k}} \rightarrow 0, \quad q_{\mathbf{k}} \rightarrow 0, \quad \text { as }|\mathbf{k}| \rightarrow \infty,
$$

where

$$
q_{\mathbf{k}}=\sum_{\mathbf{l}, \mathbf{m} \in Z^{d}} q_{\mathbf{k}, \mathbf{l}, \mathbf{m}}
$$

The data for the equations consists of a time dependent forcing $\gamma=\left\{\gamma_{\mathbf{k}}(t): k \in \mathbf{Z}^{d}, t \geqslant 0\right\}$ and an initial condition $\chi(0)=\left\{\chi_{\mathbf{k}}(0): \mathbf{k} \in \mathbf{Z}^{d}\right\}$. We consider the above system in its mild formulation, that is for given data we look for measurable $t \mapsto \chi_{\mathbf{k}}(t) \in \mathbf{C}^{r}$ satisfying, for $\mathbf{k} \in \mathbf{Z}^{d}$,

$$
\chi_{\mathbf{k}}(t)=\mathrm{e}^{-\lambda_{\mathbf{k}} t} \chi_{\mathbf{k}}(0)+\int_{0}^{t} \lambda_{\mathbf{k}} \mathrm{e}^{-\lambda_{\mathbf{k}}(t-s)}\left[C_{R} p_{\mathbf{k}} \chi_{\mathbf{k}}(s)+C_{B} \sum_{\mathbf{l}, \mathbf{m} \in \mathbf{Z}^{d}} q_{\mathbf{k}, \mathbf{l}, \mathbf{m}} B_{\mathbf{k}, \mathbf{l}, \mathbf{m}}\left(\chi_{\mathbf{l}}(s), \chi_{\mathbf{m}}(s)\right)+d_{\mathbf{k}} \gamma_{\mathbf{k}}(s)\right] \mathrm{d} s .
$$

Note that we need some regularity of $\chi_{\mathbf{k}}$, in order to make (2.4) well defined.

Remark 2.1. The abstract setting appears quite demanding in terms of the number and complexity of assumptions to be checked. The key assumption to verify is (2.3). However the examples provided below will show that is possible to get recast interesting examples into this framework, by simply exploiting the known characteristics of their non-linear operators. Moreover, we give in Section 2.5 a sufficient criterion which is easy to check for the underlying PDE.

Remark 2.2. There is considerable flexibility when choosing the constants in the ODE system (2.1). For example, we can adjust the probabilities $p_{\mathbf{k}}, q_{\mathbf{k}, \mathbf{l}, \mathbf{m}}$, and $d_{\mathbf{k}}$ by adjusting the constants $C_{B}, C_{R}$ and considering modified forcing data $\gamma$. In particular, in an equation where the probabilities do not add up to 1 in (2.2), it is always possible to adjust $d_{\mathbf{k}}$ and the forcing data so that this constraint holds. Similarly, an equation with $C_{R}$ and $C_{B}$ replaced by bounded functions of $\mathbf{k}$ can be recast into the form (2.1) by forcing the $\mathbf{k}$ dependence into the probabilities $p_{\mathbf{k}}, q_{\mathbf{k}}$, and $d_{\mathbf{k}}$. 


\subsection{The d-dimensional Burgers equations}

Consider solutions $u(t, x) \in \mathbf{R}^{d}$, for $t \geqslant 0$ and $x \in[0,2 \pi)^{d}$ to the Burgers system

$$
\left\{\begin{array}{l}
\partial_{t} u-\Delta u+(u \cdot \nabla) u=f \\
u(0)=u^{0}
\end{array}\right.
$$

with periodic boundary conditions, where $f$ is an external forcing. We restrict ourselves to periodic boundary conditions, as the non-linearity is easy to compute in the Fourier basis.

If we expand the solution

$$
u(t, x)=\sum_{\mathbf{k} \in \mathbf{Z}^{d}} u_{\mathbf{k}}(t) \mathrm{e}^{i \mathbf{k} \cdot x},
$$

the equation reads in the Fourier coefficients as

$$
\dot{u}_{\mathbf{k}}=-|\mathbf{k}|^{2} u_{\mathbf{k}}-i \sum_{\mathbf{l}+\mathbf{m}=\mathbf{k}}\left(u_{\mathbf{l}} \cdot \mathbf{m}\right) u_{\mathbf{m}}+f_{\mathbf{k}} .
$$

The sum is over all $\mathbf{l}, \mathbf{m} \in \mathbf{Z}^{d}$ satisfying $\mathbf{l}+\mathbf{m}=\mathbf{k}$. Define a weight function $w_{\mathbf{k}}=1 \vee|\mathbf{k}|^{\gamma}$, where $\gamma>0$ will be chosen shortly, and set $\chi_{\mathbf{k}}=w_{\mathbf{k}} u_{\mathbf{k}}$. Then

$$
\left\{\begin{array}{l}
\dot{\chi}_{\mathbf{k}}=-|\mathbf{k}|^{2} \chi_{\mathbf{k}}-i \sum_{\mathbf{l}+\mathbf{m}=\mathbf{k}} \frac{|\mathbf{m}| w_{\mathbf{k}}}{w_{\mathbf{m}} w_{\mathbf{l}}}\left(\chi_{\mathbf{l}} \cdot \frac{\mathbf{m}}{|\mathbf{m}|}\right) \chi_{\mathbf{m}}+f_{\mathbf{k}} w_{\mathbf{k}}, \\
\chi_{\mathbf{k}}(0)=u_{\mathbf{k}}(0) w_{\mathbf{k}}
\end{array}\right.
$$

Note that the mode $u_{\mathbf{0}}$ has no linear dissipation. Below we will add and subtract $\lambda_{\mathbf{0}} \chi_{\mathbf{0}}$ to the equation for $\chi_{\mathbf{0}}$, which introduces a linear instability but which allows us to write the equation in our desired abstract form. We note that in dimension $d=1$ this trick is unnecessary: the equations for the zeroth mode decouples, in that it simplifies to $\dot{u}_{\mathbf{0}}=f_{\mathbf{0}}$, and it is then possible to reduce the problem to the case $f_{\mathbf{0}}=u_{\mathbf{0}}=0$.

We now show one way to recast (2.6) into the abstract form (2.1). For given $C_{R}, C_{B}, \lambda_{\mathbf{0}}>0$ we define

$$
\begin{aligned}
& \lambda_{\mathbf{k}}=\left\{\begin{array}{ll}
|\mathbf{k}|^{2}, & \mathbf{k} \neq \mathbf{0}, \\
\lambda_{\mathbf{0}}, & \mathbf{k}=\mathbf{0},
\end{array} \quad p_{\mathbf{k}}= \begin{cases}0, & \mathbf{k} \neq \mathbf{0}, \\
C_{R}^{-1}, & \mathbf{k}=\mathbf{0},\end{cases} \right. \\
& q_{\mathbf{k}, \mathbf{l}, \mathbf{m}}=C_{B}^{-1} \frac{|\mathbf{m}| w_{\mathbf{k}}}{\lambda_{\mathbf{k}} w_{\mathbf{l}} w_{\mathbf{m}}},
\end{aligned}
$$

whenever $\mathbf{l}+\mathbf{m}=\mathbf{k}$ (and zero otherwise). Lemma 2.3 below ensures, provided we choose $\gamma>\max \left\{\frac{d+1}{2}, d-1\right\}$, that $q_{\mathbf{k}}=\sum_{\mathbf{l}, \mathbf{m}} q_{\mathbf{k}, \mathbf{l}, \mathbf{m}}<\infty$ and that $q_{\mathbf{k}} \rightarrow 0$ as $|\mathbf{k}| \rightarrow \infty$. Thus by taking $C_{B}, C_{R}$ sufficiently large we have that $p_{\mathbf{k}}+q_{\mathbf{k}}<1$ and it remains only to define $d_{\mathbf{k}}=1-p_{\mathbf{k}}-q_{\mathbf{k}}$ and $\gamma_{\mathbf{k}}=\left(f_{\mathbf{k}} w_{\mathbf{k}} / \lambda_{\mathbf{k}} d_{\mathbf{k}}\right)$ for $\mathbf{k} \in \mathbf{Z}^{d}$. Again, there is considerable flexibility in these choices.

Lemma 2.3. For all $\gamma_{1}, \gamma_{2}>0$ with $\gamma_{1}+\gamma_{2}>d$ there exists $C=C\left(\gamma_{1}, \gamma_{2}\right)<\infty$ so that, for all $\mathbf{k} \in \mathbf{Z}^{d}$, with $\mathbf{k} \neq \mathbf{0}$,

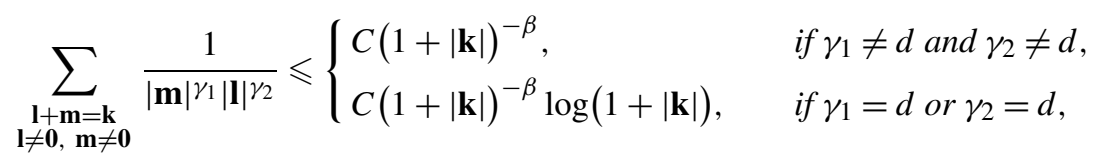

where $\beta=\min \left\{\gamma_{1}, \gamma_{2}, \gamma_{1}+\gamma_{2}-d\right\}$ and the sum is over all indices $\mathbf{l}, \mathbf{m}$ in $\mathbf{Z}^{d}$ satisfying the given constraints.

One way to prove this lemma, whose proof is omitted, is to compare above and below by suitable continuous integrals.

\subsection{Two-dimensional Navier-Stokes equations}

We briefly treat the two-dimensional Navier-Stokes in its vorticity formulation, since this will be used in Section 4 as an example where the comparison equation yields exact statements about the integrability of the stochastic representation. 
In dimension $d=2$ the vorticity $\xi=\operatorname{curl} u$ is a scalar and satisfies, on the torus $[0,2 \pi)^{2}$ and with periodic boundary conditions,

$$
\left\{\begin{array}{l}
\partial_{t} \xi-\Delta \xi+(u \cdot \nabla) \xi=f \\
\xi(0)=\xi^{0}
\end{array}\right.
$$

where $u$ is the solution to the Navier-Stokes equations. The Fourier coefficients satisfy the following system,

$$
\dot{\xi}_{\mathbf{k}}=-|\mathbf{k}|^{2} \xi_{\mathbf{k}}+\sum_{\mathbf{l}+\mathbf{m}=\mathbf{k}} \frac{\mathbf{k} \cdot \mathbf{l}^{\perp}}{|\mathbf{I}|^{2}} \xi_{\mathbf{l}} \xi_{\mathbf{m}}+f_{\mathbf{k}}
$$

where $\mathbf{I}^{\perp}=\left(l_{2},-l_{1}\right)$. For simplicity we shall assume that $f_{\mathbf{0}}=0$ and the vorticity has mean value $\xi_{\mathbf{0}}$ zero and is omitted from the system.

We then set $\chi_{\mathbf{k}}=|\mathbf{k}|^{\gamma} \xi_{\mathbf{k}}$ for some $\gamma>\frac{1}{2}$. For $C_{B}>0$ we then define

$$
\lambda_{\mathbf{k}}=|\mathbf{k}|^{2}, \quad B_{\mathbf{k}, \mathbf{l}, \mathbf{m}}\left(\chi, \chi^{\prime}\right)=\frac{\mathbf{k} \cdot \mathbf{l}^{\perp}}{\left|\mathbf{k} \cdot \mathbf{I}^{\perp}\right|} \chi \chi^{\prime}, \quad q_{\mathbf{k}, \mathbf{l}, \mathbf{m}}=C_{B}^{-1} \frac{|\mathbf{k}|^{\gamma-2}\left|\mathbf{k} \cdot \mathbf{l}^{\perp}\right|}{|\mathbf{l}|^{\gamma+2}|\mathbf{m}|^{\gamma}},
$$

for all $\mathbf{k}, \mathbf{l}, \mathbf{m} \in \mathbf{Z}^{2}$ satisfying $\mathbf{k} \cdot \mathbf{l}^{\perp} \neq 0$ and $\mathbf{l}+\mathbf{m}=\mathbf{k}$ (and zero otherwise). Lemma 2.3 ensures that $q_{\mathbf{k}}<\infty$ and that $q_{\mathbf{k}} \rightarrow 0$ as $|\mathbf{k}| \rightarrow \infty$. Taking $C_{B}$ large enough we have that $q_{\mathbf{k}}<1$ (note that here we may take $p_{\mathbf{k}}=0$ ). So the recasting is complete if we define $\gamma_{\mathbf{k}}=\left(|\mathbf{k}|^{\gamma-2} / d_{\mathbf{k}}\right) f_{\mathbf{k}}$.

\subsection{A surface growth equation}

This final example illustrates the change in weights needed for a higher order equation and the need to consider linear instabilities. In particular, the linear operator does not generate a diffusion. Therefore, the Fourier transform is necessary for the stochastic representation. Consider the following scalar equation arising in some models for surface growth,

$$
\partial_{t} u=-a_{1} \Delta^{2} u-a_{2} \Delta u-a_{3} \Delta|\nabla u|^{2}+a_{4}|\nabla u|^{2}+f,
$$

with periodic boundary conditions on $[0,2 \pi)^{d}$, with $d=1,2$ and $a_{i}>0$ for $i=1,2,3$. As for Navier-Stokes, there remain basic unresolved questions on existence and uniqueness. See Raible et al. [17] for the derivation of the model, and Blömker et al. [7] for a rigorous mathematical treatment using PDE techniques. For simplicity, we assume $a_{4}=0$ and that the mean value $\int u(t, x) \mathrm{d} x$ is zero, allowing us to omit the coefficient $u_{\mathbf{0}}$.

The equation for the Fourier coefficients is given by

$$
\dot{u}_{\mathbf{k}}=-a_{1}|\mathbf{k}|^{4} u_{\mathbf{k}}+a_{2}|\mathbf{k}|^{2} u_{\mathbf{k}}+a_{3}|\mathbf{k}|^{2} \sum_{\mathbf{l}+\mathbf{m}=\mathbf{k}}(\mathbf{l} \cdot \mathbf{m}) u_{\mathbf{l}} u_{\mathbf{m}}+f_{\mathbf{k}} .
$$

The $\mathbf{k}$ dependence in the term $+C_{R} p_{\mathbf{k}} \chi_{\mathbf{k}}$ in (2.1) is exploited in this example to allow for the term $a_{2} \Delta u$, which corresponds to the physically important linear instability of the equation. We set $\chi_{\mathbf{k}}=|\mathbf{k}|^{\gamma} u_{\mathbf{k}}$ for $\gamma>0$, with $\gamma>\max \{d, 1+d / 2\}$, and then choose, for all $\mathbf{k} \neq 0$,

$$
\lambda_{\mathbf{k}}=a_{1}|\mathbf{k}|^{4}, \quad p_{\mathbf{k}}=\frac{a_{2}}{a_{1}} C_{R}^{-1}|\mathbf{k}|^{\gamma-2}
$$

and

$$
B_{\mathbf{k}, \mathbf{l}, \mathbf{m}}\left(\chi, \chi^{\prime}\right)=\frac{\mathbf{l} \cdot \mathbf{m}}{|\mathbf{l} \cdot \mathbf{m}|} \chi \chi^{\prime}, \quad q_{\mathbf{k}, \mathbf{l}, \mathbf{m}}=C_{B}^{-1} \frac{a_{3}|\mathbf{k}|^{\gamma-2}|\mathbf{l} \cdot \mathbf{m}|}{a_{1}|\mathbf{I}|^{\gamma}|\mathbf{m}|^{\gamma}}
$$

with $B_{\mathbf{k}, \mathbf{l}, \mathbf{m}}$ and $q_{\mathbf{k}, \mathbf{l}, \mathbf{m}}$ equal to zero if $\mathbf{l} \cdot \mathbf{m}=0$ or $\mathbf{l}+\mathbf{m} \neq \mathbf{k}$. Lemma 2.3 guarantees that $p_{\mathbf{k}}+q_{\mathbf{k}}<1$ when $C_{B}, C_{R}$ are taken large enough and we can define

$$
d_{\mathbf{k}}=1-q_{\mathbf{k}}-p_{\mathbf{k}} \quad \text { and } \quad \gamma_{\mathbf{k}}=\frac{|\mathbf{k}|^{\gamma-4}}{a_{1} d_{\mathbf{k}}} f_{\mathbf{k}}
$$

to obtain a system in the form (2.1). 


\subsection{Extensions}

It is clear that the framework could be modified to allow several possible extensions, for example equations with other boundary conditions, or with polynomial non-linearities (or even analytic non-linearities, see Athreya and Tribe [2]), or with non-linear multiplicative forcing terms.

As an example, we indicate a fairly simple criterion that ensures the existence of the stochastic representation. It is easily checkable from the data of the PDE and covers equations with a variety of boundary conditions. Moreover, we give an explicit example with Dirichlet boundary conditions, to show that this is as simple as the periodic case.

\subsubsection{A general criterion}

Consider for simplicity an equation of the type

$$
\partial_{t} u=A u+\mathcal{F}(u, u),
$$

where $A$ is a self-adjoint differential operator in a Hilbert space $H$ with a complete set of normalised eigenfunctions $\left(e_{k}\right)_{k \in \mathbf{N}}$ and corresponding eigenvalues $\left(\lambda_{k}\right)_{k \in \mathbf{N}}$, and some bi-linear operator $\mathcal{F}$. Thus, using $u=\sum_{k=1}^{\infty} u_{k} e_{k}$ we derive

$$
\partial_{t} u_{k}=\lambda_{k} u_{k}+\sum_{l, m=1}^{\infty} u_{l} u_{m}\left\langle\mathcal{F}\left(e_{l}, e_{m}\right), e_{k}\right\rangle_{H} .
$$

We suppose, for simplicity of presentation, that $A$ is positive, i.e. $\lambda_{k}>0$. Assume that there is a $\delta>0$ such that

$$
\sum_{k=1}^{\infty} \lambda_{k}^{-\delta}<\infty
$$

In particular, $\lambda_{\mathbf{k}} \rightarrow \infty$ as $k \rightarrow \infty$. Finally, assume the following crucial property on the non-linear operator:

$\mathcal{F}$ is a continuous map from $D\left(A^{\alpha-\delta}\right) \times D\left(A^{\alpha-\delta}\right)$ to $D\left(A^{\alpha-1-\varepsilon}\right)$,

for some $\alpha$ and $\varepsilon>0$.

Choose weights $w_{k}=C_{0} \lambda_{k}^{\alpha}$, with $C_{0}$ large enough (according to the estimates below), and set $\chi_{k}=w_{k} u_{k}$. By computations entirely similar to the ones in the previous section, we find

$$
\begin{aligned}
q_{k, l, m} & =\frac{w_{k}}{\lambda_{k} w_{l} w_{m}}\left\langle\mathcal{F}\left(e_{l}, e_{m}\right), e_{k}\right\rangle_{H} \\
& =\frac{\lambda_{k}^{-\varepsilon}}{C_{0} \lambda_{l}^{\delta} \lambda_{m}^{\delta}}\left\langle A^{\alpha-1+\varepsilon} \mathcal{F}\left(A^{\delta-\alpha} e_{l}, A^{\delta-\alpha} e_{m}\right), e_{k}\right\rangle_{H},
\end{aligned}
$$

and, by condition (2.9), it follows that $q_{k}=\sum_{l, m} q_{k, l, m} \in[0,1)$, provided $C_{0}$ is large enough, and $q_{k} \rightarrow 0$ as $k \rightarrow \infty$. Thus the crucial condition (2.3) is fulfilled.

\subsubsection{A specific example}

Conditions (2.8) and (2.9) are quite easy to check in concrete examples. For example, for Navier-Stokes equations (in both dimensions 2 and 3), condition (2.8) follows from well-known results concerning the growth of eigenvalues for the Laplacian, while condition (2.9) follows from Lemma 2.1 of Temam [20].

The conditions of the previous section are by no means optimal and can be easily improved once the non-linearity is explicitly given. We can rely on cancellations yielding $q_{k, l, m}=0$ for many indices. Consider for example the 1D Burgers equation with Dirichlet boundary condition on $[0, \pi]$. Here $e_{k}=\sin (k x), \lambda_{k}=k^{2}$ and $\mathcal{F}(u, v)=\frac{1}{2}\left(u \cdot \partial_{x} v+\right.$ $\left.\partial_{x} u \cdot v\right)$. It is easy to verify that

$$
\mathcal{F}\left(e_{l}, e_{m}\right)=\left(\frac{l+m}{4} e_{l+m}-\frac{|l-m|}{4} e_{|l-m|}\right)
$$

so that we can directly compute

$$
q_{k}=c \frac{w_{k}}{k}\left(\sum_{l=1}^{\infty} \frac{1}{w_{l} w_{k+l}}+2 \sum_{l=1}^{k-1} \frac{1}{w_{l} w_{k-l}}\right),
$$


up to some normalisation constant $c>0$, so that for $w_{k}=C_{0} \lambda_{k}^{\alpha / 2}=C_{0} k^{\alpha}$, with $\alpha>1$ and $C_{0}$ large, we have, with computations similar to those of Lemma $2.3, q_{k} \in[0,1)$ and $q_{k} \rightarrow 0$ as $k \rightarrow \infty$. Again (2.3) is fulfilled.

\section{The branching particle representation formula}

\subsection{Existence and uniqueness}

Denote by $\|a\|_{\infty}=\sup _{\mathbf{k} \in \mathbf{Z}^{d}}\left|a_{\mathbf{k}}\right|$ the norm of bounded families $\left(a_{\mathbf{k}}\right)_{\mathbf{k} \in \mathbf{Z}^{d}}$ of elements of $\mathbf{C}^{r}$, with $\left|a_{\mathbf{k}}\right|=\sqrt{a_{\mathbf{k}} \cdot a_{\mathbf{k}}^{*}}$ the norm in $\mathbf{C}^{r}$. We start by giving a short theorem, whose proof is purely deterministic, which ensures that there is a unique local solution to (2.1). The proof is a rather standard application of the Banach fixed point theorem, and the result will be improved in Section 4 using probabilistic tools.

Theorem 3.1 (Unique local existence). Assume that

$$
\chi(0) \in \ell^{\infty}\left(\mathbf{C}^{r}\right), \quad \gamma \in L^{\infty}\left([0, T], \ell^{\infty}\left(\mathbf{C}^{r}\right)\right) \quad \text { for all } T>0 .
$$

Then there exists a time $T_{0}>0$, depending only on $\chi(0), \gamma$, and the constants appearing in the equation, such that the mild formulation (2.4) has a unique solution $\chi \in L_{\mathrm{loc}}^{\infty}\left(\left[0, T_{0}\right),\left(\mathbf{C}^{r}\right)^{\mathbf{Z}^{d}}\right)$.

Moreover, we have either $T_{0}=\infty$ or $\|\chi(t)\|_{\infty} \rightarrow \infty$ as $t \rightarrow T_{0}$. Finally, if the functions $t \mapsto \gamma_{\mathbf{k}}(t)$ are $C^{k}$, then $t \mapsto \chi_{\mathbf{k}}(t)$ are $C^{k+1}$ in time and solve Eq. (2.1).

Proof. Let $B$ be a ball of radius $R>0$ centred at the constant function with value $\chi(0)$, in the space

$$
L^{\infty}\left(\left[0, t_{\star}\right], \ell^{\infty}\left(\mathbf{C}^{r}\right)\right) .
$$

For $\chi \in B$ define $F(\chi)$ by the right-hand side of (2.4). Then for $R_{0}=R+\|\chi(0)\|_{\infty}$,

$$
\left|F(\chi)_{\mathbf{k}}(t)-\chi_{\mathbf{k}}(0)\right| \leqslant\left(\|\chi(0)\|_{\infty}+C_{R} p_{\mathbf{k}} R_{0}+C_{B} q_{\mathbf{k}} R_{0}^{2}+\|\gamma(t)\|_{\infty}\right)\left(1-\mathrm{e}^{-\lambda_{\mathbf{k}} t_{*}}\right) .
$$

If we choose $R>\|\chi(0)\|_{\infty}+\sup \|\gamma(t)\|_{\infty}$ and $t_{*}$ small enough we see that $F$ maps $B$ into itself. Here we have used assumption (2.3) to control the large $|\mathbf{k}| \mathbf{s}$. Moreover, if $\chi^{1}$ and $\chi^{2}$ are in $B$, then for $t \leqslant t_{*}$,

$$
\left|\left[F\left(\chi^{1}\right)-F\left(\chi^{2}\right)\right]_{\mathbf{k}}(t)\right| \leqslant\left(C_{R} p_{\mathbf{k}}+2 C_{B} R_{0} q_{\mathbf{k}}\right)\left(1-e^{-\lambda_{\mathbf{k}} t_{*}}\right) \sup _{t \leqslant t_{*}}\left\|\chi^{1}(t)-\chi^{2}(t)\right\|_{\infty} .
$$

Hence $F$ is a strict contraction in $L^{\infty}\left(\left[0, t_{\star}\right], \ell^{\infty}\left(\mathbf{C}^{r}\right)\right)$ if we choose $t_{*}$ small enough. Here we need again, for large $|\mathbf{k}|$, the assumption (2.3).

The assertion for the time $T_{0}$ follows in a standard manner by gluing together local solutions. The continuity of $t \rightarrow \chi_{\mathbf{k}}(t)$ follows from the mild form (2.4). It is even differentiable with bounded derivative. The $C^{k}$-regularity follows by differentiating (2.4) and the higher regularity follows from differentiating (2.1).

\subsection{The branching tree}

We now give a construction of the branching process that will be used to represent the solutions of (2.1). For the general theory of branching processes see [1,8]. We will label particles of the process with labels taken from the set $\ell=\bigcup_{n=0}^{\infty}\{0,1,2\}^{n}$ (cf. Fig. 1). The history of a particle $\alpha=\left(\alpha_{1}, \ldots, \alpha_{n}\right)$ can be read off by interpreting $\alpha_{j}=0$ as a regeneration, and $\alpha_{j}=1$ (or 2) as being child 1 (or 2) in a binary branching event at generation $j$ (binary branching events will correspond to quadratic terms in the underlying PDE whereas regenerations, which may also be thought of a branching events with a single offspring, will correspond to lower order linear terms).

For $\alpha \in\{0,1,2\}^{n}$ we write $|\alpha|=n$ which we call the length of the label. We write $\alpha=\emptyset$ for the single label of length zero. When $\alpha=\left(\alpha_{1}, \ldots, \alpha_{n}\right)$ we write $\left.\alpha\right|_{j}$ for the label $\left.\alpha\right|_{j}=\left(\alpha_{1}, \ldots, \alpha_{j}\right)$ of its ancestor at generation $j \in\{0,1, \ldots, n-1\}$ (and set $\alpha \mid 0=\emptyset$ ). For $i \in\{0,1,2\}$ we write $(i, \alpha)$ for the label $\left(i, \alpha_{1}, \ldots, \alpha_{n}\right)$ and $(\alpha, i)$ for the label $\left(\alpha_{1}, \ldots, \alpha_{n}, i\right)$ (or $(i, \alpha)=(\alpha, i)=(i)$ if $\alpha=\emptyset$ ). We construct the branching particle systems on a probability space equipped with the following independent families of I.I.D. variables: $\left(E_{\alpha}\right)_{\alpha \in \ell}$ exponential mean one variables (that will control the overall rates of branching and regenerating); $\left(U_{\alpha}\right)_{\alpha \in \ell}$ uniform $[0,1]$ variables (that will control 


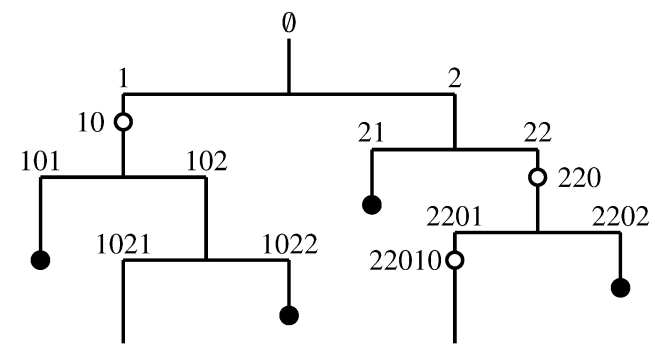

Fig. 1. A tree with branches, deaths $(\bullet)$ and regenerations $(\bigcirc)$.

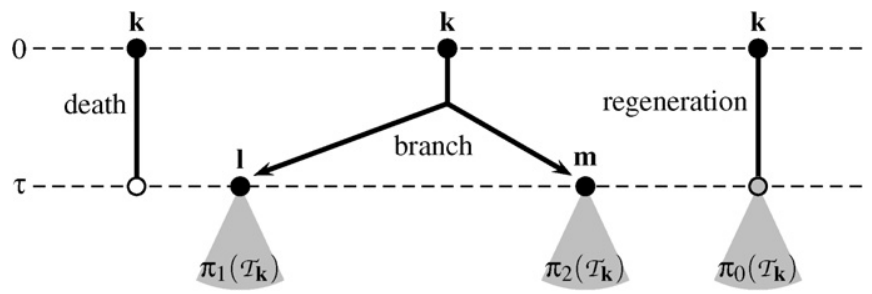

Fig. 2. The construction of the tree. At each event time $\tau$, there is a random selection between either death, regenerate or branch of two new particles to states $\mathbf{I}$ and $\mathbf{m}$ (depending on the state $\mathbf{k}$ of the parent particle).

whether a particle regenerates, branches or dies) and $\left(\left(Y_{\alpha}^{(1)}(\mathbf{k}), Y_{\alpha}^{(2)}(\mathbf{k})\right)_{\alpha \in \ell, \mathbf{k} \in \mathbf{Z}^{d}}\right.$ random variables with distribution $P\left[Y_{\alpha}^{(1)}(\mathbf{k})=\mathbf{l}, Y_{\alpha}^{(2)}(\mathbf{k})=\mathbf{m}\right]=q_{\mathbf{k}, \mathbf{l}, \mathbf{m}}$ (which will control the positions of the two offspring of a particle that branches).

We now define a system $\left(\widehat{\mathbf{K}}_{\alpha}, \tau_{\alpha}^{B}, \tau_{\alpha}^{D}\right)_{\alpha \in \ell}$ of particle positions, birth and death times, inductively over the length $n=|\alpha|$ of the labels. Fix $\mathbf{k} \in \mathbf{Z}^{d}$ and set $\widehat{\mathbf{K}}_{\emptyset}=\mathbf{k}, \tau_{\emptyset}^{B}=0$ and $\tau_{\emptyset}^{D}=\lambda_{\mathbf{k}}^{-1} E_{\emptyset}$. Assume that the positions, birth and death times have been defined for $|\alpha| \leqslant n$. Then, for $\alpha$ of length $n+1$, define birth and death times

$$
\tau_{\alpha}^{B}=\tau_{\alpha \mid n}^{D}, \quad \tau_{\alpha}^{D}=\tau_{\alpha}^{B}+\lambda_{\widehat{\mathbf{K}}_{\alpha \mid n}}^{-1} E_{\alpha},
$$

and the particle positions

$$
\widehat{\mathbf{K}}_{\alpha}= \begin{cases}\widehat{\mathbf{K}}_{\alpha \mid n}, & \alpha_{n+1}=0, \\ Y_{\alpha}^{(1)}\left(\widehat{\mathbf{K}}_{\alpha \mid n}\right), & \alpha_{n+1}=1, \\ Y_{\alpha}^{(2)}\left(\widehat{\mathbf{K}}_{\alpha \mid n}\right), & \alpha_{n+1}=2 .\end{cases}
$$

This defines a complete tree of all possible branching and regenerating particles rooted at $\mathbf{k}$. In the desired evolution the particles will choose whether to regenerate, branch or die according to the probabilities $p_{\mathbf{k}}, q_{\mathbf{k}}, d_{\mathbf{k}}$.

We now define indicator variables $\left(I_{\alpha}\right)_{\alpha \in \ell}$ to decide whether a particular branch has survived. Define $I_{\emptyset}=1$ and, for $\alpha$ of length $n+1$,

$$
I_{\alpha}= \begin{cases}1 & \text { if } \alpha_{n+1}=0 \text { and } U_{\alpha} \in\left[0, p_{\widehat{\mathbf{K}}_{\alpha \mid n}}\right] \\ 1 & \text { if } \alpha_{n+1} \in\{1,2\} \text { and } U_{\alpha} \in\left[1-q_{\widehat{\mathbf{K}}_{\alpha \mid n}}, 1\right], \\ 0 & \text { otherwise. }\end{cases}
$$

Fix an isolated cemetery state $\Delta$ and define, for $|\alpha|=n$,

$$
\mathbf{K}_{\alpha}= \begin{cases}\widehat{\mathbf{K}}_{\alpha} & \text { if } \prod_{j=1}^{n} I_{\alpha \mid j}=1, \\ \Delta & \text { otherwise. }\end{cases}
$$

The collection $\mathcal{T}_{\mathbf{k}}=\left(\mathbf{K}_{\alpha}, \tau_{\alpha}^{B}, \tau_{\alpha}^{D}\right)_{\alpha \in \ell}$ now defines our branching tree rooted at $\mathbf{k}$. It lives in the space defined by

$$
\mathcal{T}=\left(\left(\mathbf{Z}^{d} \cup\{\Delta\}\right) \times[0, \infty) \times[0, \infty)\right)^{\ell} .
$$

We denote the law of $\mathcal{T}_{\mathbf{k}}$ on $\mathcal{T}$ by $P_{\mathbf{k}}$.

The descendants of any one particle in the tree form a new tree. To make this precise we define shift maps $\pi_{i}: \mathcal{T} \rightarrow \mathcal{T}$, for $i=0,1,2$ as follows (cf. Fig. 2). For $\mathcal{T}=\left(\mathbf{k}_{\alpha}, s_{\alpha}, t_{\alpha}\right)_{\alpha \in \ell} \in \mathcal{T}$ we define a new tree $\pi_{i}(\mathcal{T})$ by

$$
\pi_{i}(\mathcal{T})=\left(\mathbf{k}_{(i, \alpha)}, s_{(i, \alpha)}-t_{\varnothing}, t_{(i, \alpha)}-t_{\varnothing}\right)_{\alpha \in \ell} .
$$


The tree $\pi_{i}(\mathcal{T})$ is meant to be the tree of descendants of the particle labelled $(i)$, with their birth and death times shifted so that particle $(i)$ is born at time $t=0$. The construction of the branching particle system from I.I.D. families implies the following lemma.

Lemma 3.2. Let $\mathcal{T}_{\mathbf{k}}=\left(\mathbf{K}_{\alpha}, \tau_{\alpha}^{B}, \tau_{\alpha}^{D}\right)_{\alpha \in \ell}$ have law $\mathrm{P}_{\mathbf{k}}$. Then

(1) conditional on $\left\{\tau_{\emptyset}^{D} \in d s, \mathbf{K}_{(0)}=\mathbf{k}\right\}$ the tree $\pi_{0}\left(\mathcal{T}_{\mathbf{k}}\right)$ has the law $\mathbf{P}_{\mathbf{k}}$;

(2) conditional on $\left\{\tau_{\emptyset}^{D} \in d s, \mathbf{K}_{(1)}=\mathbf{m}, \mathbf{K}_{(2)}=\mathbf{l}\right\}$ the trees $\pi_{1}\left(\mathcal{T}_{\mathbf{k}}\right)$ and $\pi_{2}\left(\mathcal{T}_{\mathbf{k}}\right)$ are independent and have laws $\mathbf{P}_{\mathbf{m}}$ and $\mathrm{P}_{\mathrm{l}}$.

We want to ensure that the tree has only finitely many branches before time $t$. Define $N_{[0, t]}: \mathcal{T} \rightarrow \mathbf{N}$ by $N_{[0, t]}(\mathcal{T})=$ $\left|\left\{\alpha \in \ell: s_{\alpha} \leqslant t\right\}\right|$, that is the cardinality of the set of particles born before time $t$.

Lemma 3.3. Under $\mathrm{P}_{\mathbf{k}}$ the variables $N_{[0, t]}$ are almost surely finite for all $t \geqslant 0$.

Proof. Let $P_{\mathbf{k}}(t)=\mathrm{P}_{\mathbf{k}}\left[N_{[0, t]}<\infty\right]$. By conditioning on the values of $\tau_{\emptyset}^{D}, \mathbf{K}_{(0)}, \mathbf{K}_{(1)}, \mathbf{K}_{(2)}$ and using Lemma 3.2,

$$
P_{\mathbf{k}}(t)=e^{-\lambda_{\mathbf{k}} t}+\int_{0}^{t} \lambda_{\mathbf{k}} \mathrm{e}^{-\lambda_{\mathbf{k}}(t-s)}\left[p_{\mathbf{k}} P_{\mathbf{k}}(s)+\sum_{\mathbf{l}, \mathbf{m} \in \mathbf{Z}^{d}} q_{\mathbf{k}, \mathbf{l}, \mathbf{m}} P_{\mathbf{l}}(s) P_{\mathbf{m}}(s)+d_{\mathbf{k}}\right] \mathrm{d} s .
$$

Hence, $\left(P_{\mathbf{k}}(t): \mathbf{k} \in \mathbf{Z}^{d}, t \geqslant 0\right)$ is a bounded, real-valued solution to Eq. (2.1) with forcing $\gamma \equiv 1$ and bilinear operators $B_{\mathbf{k}, \mathbf{l}, \mathbf{m}}\left(\chi, \chi^{\prime}\right)=\chi \chi^{\prime}$. By Theorem 3.1, there is only one solution, namely $P_{\mathbf{k}}(t)=1$ for all $\mathbf{k}, t$.

A simple criterion that ensures that the branching process becomes extinct with probability one, that is $\mathbf{K}_{\alpha}=\Delta$ for all large $|\alpha|$, is that

$$
q_{\mathbf{k}} \leqslant d_{\mathbf{k}} \quad \text { and } \quad p_{\mathbf{k}}<1 \quad \text { for all } \mathbf{k} \in \mathbf{Z}^{d} .
$$

This is easy to fulfil by modifying (enlarging) the constants $C_{B}$ and $C_{R}$ in (2.1). Indeed the number of particles alive at time $t$ is an integer valued process whose successive values, under the condition (3.1), form a sub-critical branching process. Therefore it eventually reaches zero. The number of values $\mathbf{k} \in \mathbf{Z}^{d}$ taken by particles before this extinction is almost surely finite. The conditions that $\lambda_{\mathbf{k}}>0$ and $p_{\mathbf{k}}<1$ ensure that the extinction time for the branching particle system is almost surely finite. Note that, as explained in Remark 2.2, we can always choose the system (2.1) in such a way that (3.1) holds.

\subsection{The evaluation along the tree}

We now fix a forcing function $\gamma$ and an initial condition $\chi(0)$. We wish to define evaluation maps $R_{t}: \mathcal{T} \rightarrow \mathbf{C}^{r}$ for $t \geqslant 0$, which will depend on $\gamma$ and $\chi(0)$. These will satisfy a recursive property that allows them to be calculated backwards along the tree.

For the sake of simplicity, we introduce the following abbreviations: given a branching tree $\mathcal{T}=\left(\mathbf{k}_{\alpha}, s_{\alpha}, t_{\alpha}\right)_{\alpha \in \ell}$ and a particle labelled $\alpha \in \ell$, with $\mathbf{k}_{\alpha} \neq \Delta$, we say that the particle has a

death: if $\mathbf{k}_{(\alpha, 0)}=\mathbf{k}_{(\alpha, 1)}=\mathbf{k}_{(\alpha, 2)}=\Delta$,

regeneration: if $\mathbf{k}_{(\alpha, 0)} \neq \Delta$ and $\mathbf{k}_{(\alpha, 1)}, \mathbf{k}_{(\alpha, 2)}=\Delta$,

branch: if $\mathbf{k}_{(\alpha, 0)}=\Delta$ and $\mathbf{k}_{(\alpha, 1)}, \mathbf{k}_{(\alpha, 2)} \neq \Delta$.

Under each probability $\mathrm{P}_{\mathbf{k}}$, every particle $\alpha$ for which $\mathbf{K}_{\alpha} \neq \Delta$ must do exactly one of the above three possibilities. 
Lemma 3.4. There exists a family of maps $R_{t}: \mathcal{T} \rightarrow \mathbf{C}^{r}$, for $t \geqslant 0$, satisfying, when $N_{[0, t]}(\mathcal{T})<\infty$, the implicit formula

$$
R_{t}(\mathcal{T})= \begin{cases}\chi_{\mathbf{k}_{\emptyset}}(0), & t_{\emptyset} \geqslant t, \\ \gamma_{\mathbf{k}_{\emptyset}}\left(t-t_{\emptyset}\right), & t_{\emptyset}<t, \text { death at } \emptyset, \\ C_{R} R_{t-t_{\emptyset}}\left(\pi_{0}(\mathcal{T})\right), & t_{\emptyset}<t, \text { regen. at } \emptyset, \\ C_{B} B_{\mathbf{k}_{\emptyset}, \mathbf{k}_{(1)}, \mathbf{k}_{(2)}}\left(R_{t-t_{\emptyset}}\left(\pi_{1}(\mathcal{T})\right), R_{t-t_{\emptyset}}\left(\pi_{2}(\mathcal{T})\right)\right), & t_{\emptyset}<t, \text { branch at } \emptyset .\end{cases}
$$

Proof. Informally, since the tree is finite when $N_{[0, t]}<\infty$ the value of $R_{t}(\mathcal{T})$ can be calculated backwards along the tree, starting at time $s=t$ and working back to time $s=0$ : evaluate the initial condition $\chi(0)$ at any particles that are alive at time $t$, evaluate the forcing function $\gamma(s)$ at any particle that dies at time $s<t$, and apply the bilinear operators at the times of branching events.

For a careful proof one can define a sequence of approximations $R_{n, t}^{(a p p r)}$ in the following way:

$$
R_{1, t}^{\text {(apprx })}(\mathcal{T})= \begin{cases}\chi_{\mathbf{k}_{\emptyset}}(0) & \text { if } t_{\emptyset} \geqslant t, \\ \gamma_{\mathbf{k}_{\emptyset}}\left(t-t_{\emptyset}\right) & \text { if } t_{\emptyset}<t, \text { death at } \emptyset, \\ 1 & \text { otherwise, }\end{cases}
$$

and $R_{n+1, t}^{(\text {apprx }}(\mathcal{T})$ is given by

$$
\begin{cases}\chi_{\mathbf{k}_{\emptyset}}(0), & t_{\emptyset} \geqslant t, \\ \gamma_{\mathbf{k}_{\emptyset}}\left(t-t_{\emptyset}\right), & t_{\emptyset}<t, \text { death at } \emptyset, \\ C_{R} R_{n, t-t_{\emptyset}}^{(\text {appry }}\left(\pi_{0}(\mathcal{T})\right), & t_{\emptyset}<t, \text { regener. at } \emptyset, \\ C_{B} B_{\mathbf{k}_{\emptyset}, \mathbf{k}_{(1)}, \mathbf{k}_{(2)}}\left(R_{n, t-t_{\emptyset}}^{(\text {apprx })}\left(\pi_{1}(\mathcal{T})\right), R_{n, t-t_{\emptyset}}^{(\text {apprx })}\left(\pi_{2}(\mathcal{T})\right)\right), & t_{\emptyset}<t, \text { branch at } \emptyset .\end{cases}
$$

If $N_{[0, t]}<\infty$ then only finitely many iterations are needed and $R_{t}(\mathcal{T})=R_{n, t}^{(\text {apprx })}(\mathcal{T})$ for all large $n$.

In some cases the evaluation can be written more explicitly. Let $F(t)$ (respectively $B(t)$ ) be the number of particles that have regenerated (respectively branched) before time $t$. Let $D(t)$ be the set of labels of particles that have died strictly before time $t$.

Consider the special case where $r=1$ and that all the bilinear forms $B_{\mathbf{k}, \mathbf{l}, \mathbf{m}}$ coincide with the usual product in $\mathbf{C}$. Then the evaluation is given, almost surely under $\mathrm{P}_{\mathbf{k}}$, by

$$
R_{t}(\mathcal{T})=C_{B}^{B(t)} C_{R}^{F(t)} \prod_{\alpha \in D(t)} \gamma_{\mathbf{k}_{\alpha}}\left(t-t_{\alpha}\right) \prod_{\alpha: t \in\left[s_{\alpha}, t_{\alpha}\right)} \chi_{\mathbf{k}_{\alpha}}(0) .
$$

In the general case, we can only verify, under $\mathrm{P}_{\mathbf{k}}$, that

$$
\left|R_{t}(\mathcal{T})\right| \leqslant C_{B}^{B(t)} C_{R}^{F(t)} \prod_{\alpha \in D(t)}\left|\gamma_{\mathbf{k}_{\alpha}}\left(t-t_{\alpha}\right)\right| \prod_{\alpha: t \in\left[s_{\alpha}, t_{\alpha}\right)}\left|\chi_{\mathbf{k}_{\alpha}}(0)\right|,
$$

and that equality holds in (3.4) if $\left|B_{\mathbf{k}, \mathbf{l}, \mathbf{m}}\left(\chi, \chi^{\prime}\right)\right|=|\chi|\left|\chi^{\prime}\right|$ for all $\mathbf{k}, \mathbf{l}, \mathbf{m}$ and $\chi, \chi^{\prime}$.

\subsection{The representation formula}

Consider an initial condition $\chi(0) \in \ell^{\infty}\left(\mathbf{C}^{r}\right)$, and a forcing $\gamma \in L^{\infty}\left([0, T], \ell^{\infty}\left(\mathbf{C}^{r}\right)\right)$. The representation formula for solutions of (2.1), when the expectation exists, is given by

$$
\chi_{\mathbf{k}}(t)=\mathrm{E}_{\mathbf{k}}\left[R_{t}\right], \quad \mathbf{k} \in \mathbf{Z}^{d} .
$$

Theorem 3.5. Suppose that there exists $C=C(\gamma, \chi(0), T)<\infty$ so that

$$
\mathrm{E}_{\mathbf{k}}\left|R_{t}\right| \leqslant C \quad \text { for all } \mathbf{k} \in \mathbf{Z}^{d} \text { and all } t \in[0, T] .
$$

Then $\chi$ defined in (3.5) is the unique $L^{\infty}\left([0, T], \ell^{\infty}\left(\mathbf{C}^{r}\right)\right)$ solution of problem (2.1) for the data $\gamma, \chi(0)$. 
Proof. Note that uniqueness follows from Theorem 3.1. Fix a $\mathbf{k} \in \mathbf{Z}^{d}$. Conditioning on the values of $\tau_{\emptyset}, \mathbf{K}_{(0)}, \mathbf{K}_{(1)}$, $\mathbf{K}_{(2)}$ and using Lemma 3.2 leads immediately to the mild form of Eq. (2.4). The uniform (over $\left.\mathbf{k}\right)$ integrability is necessary to show that the sum over $\mathbf{l}, \mathbf{m}$ converges.

In the next two sections we discuss how to check the integrability assumption and what to do if it fails. We also see what happens if the solution fails to be in $\ell^{\infty}$.

\section{The comparison equation}

\subsection{The comparison equation}

The comparison equation for system (2.1) is formed by taking the norm of the data $\left|\chi_{\mathbf{k}}(0)\right|$ and $\left|\gamma_{\mathbf{k}}\right|$ as new data for the system

$$
\left\{\begin{array}{l}
\dot{\tilde{\chi}}_{\mathbf{k}}=\lambda_{\mathbf{k}}\left[-\tilde{\chi}_{\mathbf{k}}+C_{R} p_{\mathbf{k}} \tilde{\chi}_{\mathbf{k}}+C_{B} \sum_{\mathbf{l}, \mathbf{m} \in \mathbf{Z}^{d}} q_{\mathbf{k}, \mathbf{l}, \mathbf{m}} \tilde{\chi}_{\mathbf{l}} \tilde{\chi}_{\mathbf{m}}+d_{\mathbf{k}}\left|\gamma_{\mathbf{k}}\right|\right], \\
\tilde{\chi}_{\mathbf{k}}(0)=\left|\chi_{\mathbf{k}}(0)\right|,
\end{array}\right.
$$

for $\mathbf{k} \in \mathbf{Z}^{d}$. We now look for non-negative real solutions $\tilde{\chi}_{\mathbf{k}}(t)$.

We also define a modified evaluation operator $\widetilde{R}_{t}$ on $\mathcal{T}$ by the implicit formula (3.2) where we use the new data $\left|\chi_{\mathbf{k}}(0)\right|$ and $\left|\gamma_{\mathbf{k}}\right|$ and the bilinear operators are replaced by $\widetilde{B}_{\mathbf{k}, \mathbf{l}, \mathbf{m}}\left(\chi, \chi^{\prime}\right)=\chi \chi^{\prime}$, the usual product of real numbers. Then $\widetilde{R}_{t}(\mathcal{T}) \geqslant 0$ and formally we expect that

$$
\tilde{\chi}_{\mathbf{k}}(t)=\mathrm{E}_{\mathbf{k}}\left[\widetilde{R}_{t}\right], \quad \mathbf{k} \in \mathbf{Z}^{d},
$$

should solve the comparison equation.

The next theorem confirms this and shows that a finite solution to the comparison equation (4.1) is a sufficient, and sometimes necessary, condition for the tree expectations $\mathrm{E}_{\mathbf{k}}\left[R_{t}\right]$ to exist.

Theorem 4.1. If the expectations in (4.2) are finite for all $t \in[0, T]$ and $\mathbf{k} \in \mathbf{Z}^{d}$, then they define a mild solution to the comparison equation (4.1) for which $t \rightarrow \tilde{\chi}_{\mathbf{k}}(t)$ is continuous on $[0, T]$.

Conversely if there exists a finite mild solution of (4.1), that is $\tilde{\chi}_{\mathbf{k}}(t)<\infty$ for $t \in[0, T]$ and $\mathbf{k} \in \mathbf{Z}^{d}$, then the expectations in (4.2) are finite for $t \in[0, T]$, and they define the smallest positive solution of (4.1).

Finally, the comparison $\mathrm{E}_{\mathbf{k}}\left[\left|R_{t}\right|\right] \leqslant \mathrm{E}_{\mathbf{k}}\left[\widetilde{R}_{t}\right]$ holds, with equality whenever $\left|B_{\mathbf{k}, \mathbf{l}, \mathbf{m}}\left(\chi, \chi^{\prime}\right)\right|=\left|\chi \| \chi^{\prime}\right|$ for all $\mathbf{k}, \mathbf{l}, \mathbf{m}$ and $\chi, \chi^{\prime}$.

Proof. For the first claim of the theorem, condition on the values of $\tau_{\emptyset}^{D}, \mathbf{K}_{(0)}, \mathbf{K}_{(1)}, \mathbf{K}_{(2)}$ and apply Lemma 3.2 to see that the expectations $\tilde{\chi}_{\mathbf{k}}(t)=\mathrm{E}_{\mathbf{k}}\left[\widetilde{R}_{t}\right]$ satisfy the mild form of the comparison equation. Moreover the mild form of the equation shows that $\mathrm{e}^{\lambda_{\mathbf{k}} t} \tilde{\chi}_{\mathbf{k}}(t)$ is continuous and increasing in $t$. Note that the convergence of the series in the mild formulation is not a problem here, because due to positivity, we can use monotone convergence.

For the second part of the theorem, let $\tilde{\chi}$ be a mild solution of the comparison equation (4.1) in $[0, T]$ with data $\left|\chi_{\mathbf{k}}(0)\right|$ and $\left|\gamma_{\mathbf{k}}\right|$. Define a sequence of evaluations on the trees as follows: set $\widetilde{R}_{0, t}^{(\text {expl })}(\mathcal{T}, \tilde{\chi})=\tilde{\chi}_{\mathbf{k}_{\emptyset}}(t)$ and for each $n \geqslant 0$,

$$
\widetilde{R}_{n+1, t}^{(\text {expl })}(\mathcal{T}, \tilde{\chi})= \begin{cases}\left|\chi_{\mathbf{k}_{\emptyset}}(0)\right|, & t_{\emptyset} \geqslant t, \\ \left|\gamma_{\mathbf{k}_{\emptyset}}\left(t-t_{\emptyset}\right)\right|, & t_{\emptyset}<t, \text { death at } \emptyset, \\ C_{R} \widetilde{R}_{n, t-t_{\emptyset}}^{(\text {expl }}\left(\pi_{0}(\mathcal{T}), \tilde{\chi}\right), & t_{\emptyset}<t, \text { regen. at } \emptyset, \\ C_{B} \widetilde{R}_{n, t-t_{\emptyset}}^{(\text {expl }}\left(\pi_{1}(\mathcal{T}), \tilde{\chi}\right) \widetilde{R}_{n, t-t_{\emptyset}}^{(\operatorname{expl})}\left(\pi_{2}(\mathcal{T}), \tilde{\chi}\right), & t_{\emptyset}<t \text {, branch at } \emptyset .\end{cases}
$$

(In the language of next section, the evaluation $\widetilde{R}_{n, t}^{(\text {expl })}$ corresponds to a pruning of the tree after $n$ generations and the expectation $\mathrm{E}_{\mathbf{k}} \widetilde{R}_{n, t}^{(\text {expl) }}(\mathcal{T}, \tilde{\chi})$ will solve a Picard iteration scheme for (4.1).) 
Note that, upon dying, regenerating or branching, particles of length $n$ are evaluated using the true solution $\tilde{\chi}$. Inductively one checks, by conditioning on the first event, that for all $n \geqslant 0$

$$
\mathrm{E}_{\mathbf{k}}\left[\widetilde{R}_{n, t}^{(\mathrm{expl})}(\mathcal{T}, \tilde{\chi})\right]=\tilde{\chi}_{\mathbf{k}}(t)
$$

Since $N_{[0, t]}<\infty$ under $\mathrm{P}_{\mathbf{k}}$ we have that $\widetilde{R}_{n, t}^{(\text {expl) }}(\mathcal{T}) \rightarrow \widetilde{R}_{t}(\mathcal{T})$ almost surely. By Fatou's lemma and (4.4) we find that $\mathrm{E}_{\mathbf{k}}\left[\widetilde{R}_{t}\right] \leqslant \tilde{\chi}_{\mathbf{k}}(t)<\infty$.

The third claim of the theorem is immediate from the upper bound (3.4) and the fact that it is an equality under the conditions given.

Remark 4.2. Note that in the above theorem, and its corollary below, we do not insist the solutions are bounded in $\ell^{\infty}$.

The first two parts of the above theorem show that, when there exists a finite mild solution $\tilde{\chi}$ to (4.1), the function defined by $\mathrm{E}_{\mathbf{k}}\left[\widetilde{R}_{t}\right]$ is the smallest solution to (4.1) lying below $\tilde{\chi}$. Note in the case of $\ell^{\infty}$ solutions there is uniqueness of solutions, as in Theorem 3.1.

As in Le Jan and Sznitman [10], it is possible, when there exists a finite mild solution $\tilde{\chi}$ to (4.1), to show that $n \rightarrow \widetilde{R}_{n, t}^{(\text {expl) }}(\mathcal{T}, \tilde{\chi})$ is a non-negative martingale (with respect to a natural filtration along generations of the tree). Uniform integrability of this martingale would then imply that $\tilde{\chi}_{\mathbf{k}}(t)=\mathrm{E}_{\mathbf{k}}\left[\widetilde{R}_{t}\right]$.

Corollary 4.3. Under the conditions of either the first or the second part of Theorem 4.1 the expectations $\chi_{\mathbf{k}}(t)=$ $\mathrm{E}_{\mathbf{k}}\left[R_{t}\right]$ are well defined for $t \in[0, T]$ and $\mathbf{k} \in \mathbf{Z}^{d}$ and form a mild solution to (2.1). Moreover, such a solution is unique among all mild solutions $\chi^{\prime}$ such that

$$
\left|\chi_{\mathbf{k}}^{\prime}(t)\right| \leqslant \mathrm{E}_{\mathbf{k}}\left[\widetilde{R}_{t}\right], \quad \text { for all } \mathbf{k} \in \mathbf{Z}^{d}, t \in[0, T] .
$$

Proof. The expectations $\mathrm{E}_{\mathbf{k}}\left[R_{t}\right]$ are well defined by Theorem 4.1 as $\left|R_{t}\right| \leqslant \widetilde{R}_{t}$. By conditioning on the first event as before they will solve the mild equation. Note that in this case the convergence of the sums in the mild equation is ensured by the finiteness of the comparison equation.

Let $\chi^{\prime}$ be a mild solution verifying (4.5) and define a sequence of evaluations $R_{n, t}^{(\operatorname{expl})}\left(\mathcal{T}, \chi^{\prime}\right)$ for $n \in \mathbf{N}$ as in the proof of previous theorem, that is $R_{0, t}^{(\text {expl) }}(\mathcal{T})=\chi_{\mathbf{k}_{\varnothing}}^{\prime}(t)$ and, for all $n \geqslant 1, R_{n, t}^{(e x p l)}(\mathcal{T})$ is defined as in formula (4.3) with data $\chi^{\prime}$ and $\gamma$ and with products $B_{\mathbf{k}, \mathbf{k}_{(1)}, \mathbf{k}_{(2)}}$ in the place of usual product. By assumption (4.5) and an argument similar to (3.4) it follows that

$$
\left|R_{n, t}^{(\mathrm{expl})}\left(\mathcal{T}, \chi^{\prime}\right)\right| \leqslant \widetilde{R}_{n, t}^{(\mathrm{expl})}(\mathcal{T}, \tilde{\chi}),
$$

where $\tilde{\chi}_{\mathbf{k}}(t)=\mathrm{E}_{\mathbf{k}}\left[\widetilde{R}_{t}\right]$ and $R_{n, t}^{(\text {expl) }}(\mathcal{T}, \tilde{\chi})$ are taken from the proof of Theorem 4.1. Moreover, as in that proof, we can show inductively that $\chi_{\mathbf{k}}^{\prime}(t)=\mathrm{E}_{\mathbf{k}}\left[R_{n, t}^{(\mathrm{expl})}\left(\mathcal{T}, \chi^{\prime}\right)\right]$.

We next note that $R_{n, t}^{(\operatorname{expl})}\left(\mathcal{T}, \chi^{\prime}\right)=R_{t}(\mathcal{T})$ and $\widetilde{R}_{n, t}^{(\text {expl })}(\mathcal{T}, \tilde{\chi})=\widetilde{R}_{t}(\mathcal{T})$ on the set $\Omega_{n, t}=\left\{N_{[0, t]}(\mathcal{T}) \leqslant n\right\}$. Thus,

$$
\begin{aligned}
\mathrm{E}_{\mathbf{k}}\left[\widetilde{R}_{n, t}^{(\operatorname{expl})}(\mathcal{T}, \tilde{\chi}) \mathbf{1}_{\Omega_{n, t}^{c}}\right] & =\tilde{\chi}_{\mathbf{k}}(t)-\mathrm{E}_{\mathbf{k}}\left[\widetilde{R}_{n, t}^{(\operatorname{expl})}(\mathcal{T}, \tilde{\chi}) \mathbf{1}_{\Omega_{n, t}}\right] \\
& =\mathrm{E}_{\mathbf{k}}\left[\widetilde{R}_{t}\right]-\mathrm{E}_{\mathbf{k}}\left[\widetilde{R}_{t} \mathbf{1}_{\Omega_{n, t}}\right]=\mathrm{E}_{\mathbf{k}}\left[\widetilde{R}_{t} \mathbf{1}_{\Omega_{n, t}^{c}}\right],
\end{aligned}
$$

and therefore

$$
\begin{aligned}
\left|\chi_{\mathbf{k}}^{\prime}(t)-\mathrm{E}_{\mathbf{k}}\left[R_{t}\right]\right| & \leqslant \mathrm{E}_{\mathbf{k}}\left|R_{n, t}^{(\text {expl) }}\left(\mathcal{T}, \chi^{\prime}\right)-R_{t}\right|=\mathrm{E}_{\mathbf{k}}\left[\left|R_{n, t}^{(\text {expl })}\left(\mathcal{T}, \chi^{\prime}\right)-R_{t}\right| \mathbf{1}_{\Omega_{n, t}^{c}}\right] \\
& \leqslant \mathrm{E}_{\mathbf{k}}\left[\left(\widetilde{R}_{n, t}^{(\text {expl })}(\mathcal{T}, \tilde{\chi})+\widetilde{R}_{t}\right) \mathbf{1}_{\Omega_{n, t}^{c}}\right]=2 \mathrm{E}_{\mathbf{k}}\left[\widetilde{R}_{t} \mathbf{1}_{\Omega_{n, t}^{c}}\right] .
\end{aligned}
$$

Letting $n \rightarrow \infty$ we conclude that $\chi^{\prime}=\chi$, the solution given by the probabilistic representation.

\subsection{Examples}

We can remove the weights used to cast the equation into our abstract form and rewrite the comparison equation as equations for the Fourier coefficients of a scalar PDE. 
Consider the Burgers equation example discussed in Section 2.2 (similar considerations hold for Burgers with other boundary conditions, as in Section 2.5.2). Defining $\tilde{u}_{\mathbf{k}}=w_{\mathbf{k}}^{-1} \tilde{\chi}_{\mathbf{k}}$ we obtain a comparison equation of the form

$$
\begin{aligned}
& \dot{\tilde{u}}_{\mathbf{k}}=-|\mathbf{k}|^{2} \tilde{u}_{\mathbf{k}}+\sum_{\mathbf{1}+\mathbf{m}=\mathbf{k}}|\mathbf{m}| \tilde{u}_{\mathbf{l}} \tilde{u}_{\mathbf{m}}+\left|f_{\mathbf{k}}\right|, \\
& \tilde{u}_{\mathbf{k}}(0)=\left|u_{\mathbf{k}}^{0}\right|
\end{aligned}
$$

which in the space coordinates corresponds to the scalar equation

$$
\partial_{t} \tilde{u}=\Delta \tilde{u}+\tilde{u}(-\Delta)^{1 / 2} \tilde{u}+\tilde{f},
$$

where $\tilde{f}$ has Fourier coefficients $\left|f_{\mathbf{k}}\right|$. Note that this scalar comparison equation is independent of the choice of weights (called majorizing kernels in Bhattacharya et al. [4]).

For the two-dimensional Navier-Stokes equation discussed in Section 2.3 the comparison equation for $\tilde{\xi}_{\mathbf{k}}=$ $|\mathbf{k}|^{-\gamma} \tilde{\chi}_{\mathbf{k}}$ takes the form

$$
\dot{\tilde{\xi}}_{\mathbf{k}}=-|\mathbf{k}|^{2} \tilde{\xi}_{\mathbf{k}}+\sum_{\mathbf{l}+\mathbf{m}=\mathbf{k}} \frac{\left|\mathbf{k} \cdot \mathbf{l}^{\perp}\right|}{|\mathbf{l}|^{2}} \tilde{\xi}_{\mathbf{l}} \tilde{\xi}_{\mathbf{m}}+\left|f_{\mathbf{k}}\right|,
$$

which does not have a nice expression in the space variables.

For the surface equations discussed in Section 2.4 the comparison equation becomes

$$
\partial_{t} \tilde{u}=-a_{1} \Delta^{2} \tilde{u}-a_{2} \Delta \tilde{u}-\Delta\left|(-\Delta)^{1 / 2} \tilde{u}\right|^{2}+\tilde{f},
$$

where the forcing $\tilde{f}$ has Fourier coefficients $\left|f_{\mathbf{k}}\right|$.

Whenever there is a solution to these scalar comparison equations with finite Fourier coefficients we obtain the existence of mild solutions to the corresponding abstract ODEs given by the stochastic representation (3.5) This in turn is equivalent to the existence of solutions to the original PDEs with finite Fourier coefficients.

Remark 4.4. All three scalar comparison equations have quadratic growth. In Montgomery-Smith [14], a finite time blow-up has been shown for a "cheap Navier-Stokes" equation, which is entirely similar to the comparison equations given below. See also Lopez-Mimbela and Wakolbinger [12] and the references therein for the case of branching with diffusion.

In the case of the 2D Navier-Stokes, the 1D Burgers, or the surface equation, the equality in the last part of Theorem 4.1 holds. This implies that the stochastic representation $\mathrm{E}_{\mathbf{k}}\left[R_{t}\right]$ is well defined as the expectation of an integrable variable, if and only if the corresponding comparison equation has a solution with finite Fourier coefficients. In particular, the results cited above imply that, for any suitable weight, the representation will fail to exist at some finite time.

\section{The pruned approximation}

\subsection{A general approximation scheme}

The aim is to define a sequence of approximations $\chi_{\mathbf{k}}^{(n)}(t)$ to our abstract system of ODEs (2.1). These approximations will have a stochastic representation without any integrability problems.

Rather than construct a particle system with labelled particles as described in the previous section (cf. also Fig. 3), we put the modification into the evaluation operators. We claim there exists a sequence of evaluation operators $R_{n, t}: \mathcal{T} \rightarrow \mathbf{C}^{r}$ satisfying the following implicit relations on $N_{[0, t]}<\infty:$

$$
R_{0, t}(\mathcal{T})= \begin{cases}\chi_{\mathbf{k}_{\emptyset}}(0) & \text { if } t_{\emptyset} \geqslant t \\ 0 & \text { otherwise }\end{cases}
$$

and, for $n \geqslant 1, R_{n, t}(\mathcal{T})$ equals

$$
\begin{cases}\chi_{\mathbf{k}_{\emptyset}}(0), & t_{\emptyset} \geqslant t, \\ \gamma_{\mathbf{k}_{\emptyset}}\left(t-t_{\emptyset}\right), & t_{\emptyset}<t, \text { death at } \emptyset, \\ C_{R} R_{n, t-t_{\emptyset}}\left(\pi_{0}(\mathcal{T})\right), & t_{\emptyset}<t, \text { regener. at } \emptyset, \\ C_{B} B_{\mathbf{k}_{\emptyset}, \mathbf{k}_{(1)}, \mathbf{k}_{(2)}}\left(R_{n, t-t_{\emptyset}}\left(\pi_{1}(\mathcal{T})\right), R_{n-1, t-t_{\emptyset}}\left(\pi_{2}(\mathcal{T})\right)\right), & t_{\emptyset}<t \text { branch at } \emptyset .\end{cases}
$$




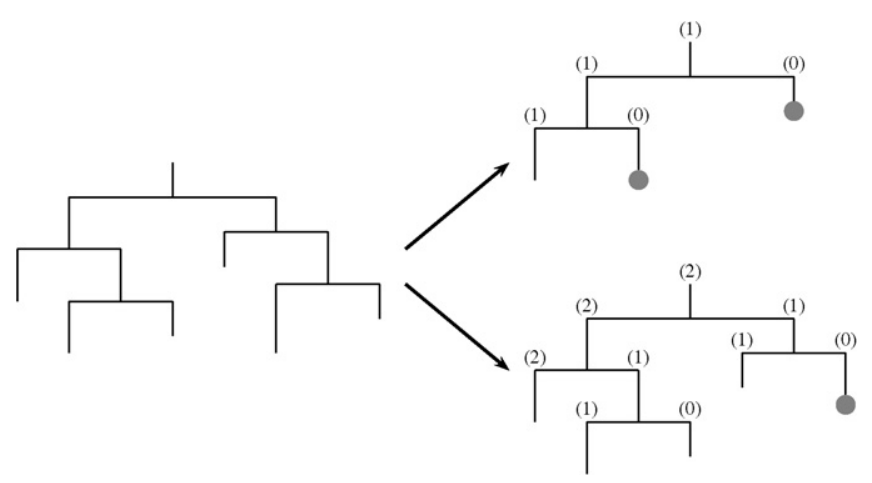

Fig. 3. The tree on the left can be pruned by starting, top right, with a particle labelled (1), or, bottom right, by a particle labelled (2). Circles mark the death via pruning. If a label larger than (2) is given to the starting particle, the tree is un-pruned.

The existence of $R_{n, t}$ can be established exactly as in Lemma 3.4. The intuitive link with the labelled particle picture in the last section is that $R_{n, t}(\mathcal{T})$ corresponds to the evaluation operator applied to the tree started at a particle with label $n$ at position $\mathbf{k}$.

The implicit relation implies that if $N_{[0, t]} \leqslant m$ then $R_{n, t}(\mathcal{T})=R_{t}(\mathcal{T})$ whenever $n \geqslant m$. Moreover when $R_{n, t}(\mathcal{T}) \neq$ $R_{t}(\mathcal{T})$ then $R_{n, t}(\mathcal{T})=0$. Thus there exist increasing sets $\Omega_{n, t} \subset \mathcal{T}$ so that

$$
R_{n, t}\left(\mathcal{T}_{\mathbf{k}}\right)=R_{t}\left(\mathcal{T}_{\mathbf{k}}\right) \mathbf{1}_{\Omega_{n, t}} \quad \text { and } \quad\left\{N_{[0, t]}<\infty\right\} \subseteq \bigcup_{n} \Omega_{n, t}
$$

We now define the stochastic representation using these modified evaluations by

$$
\chi_{\mathbf{k}}^{(n)}(t)=\mathrm{E}_{\mathbf{k}}\left[R_{n, t}\right]
$$

The fact that this expectation is always well defined is part of the following result.

Proposition 5.1. Suppose that $\chi(0) \in \ell^{\infty}\left(\mathbf{C}^{r}\right)$ and $\gamma \in L^{\infty}\left([0, T], \ell^{\infty}\left(\mathbf{C}^{r}\right)\right)$. Then the expectations in (5.2) are well defined and $\chi_{\mathbf{k}}^{(n)}(t)$ are the unique $L^{\infty}\left([0, T], \ell^{\infty}\left(\mathbf{C}^{r}\right)\right)$ mild solution to the following approximation scheme

$$
\begin{aligned}
& \dot{\chi}_{\mathbf{k}}^{(0)}=-\lambda_{\mathbf{k}} \chi_{\mathbf{k}}^{(0)}, \\
& \dot{\chi}_{\mathbf{k}}^{(n)}=\lambda_{\mathbf{k}}\left[-\chi_{\mathbf{k}}^{(n)}+C_{R} p_{\mathbf{k}} \chi_{\mathbf{k}}^{(n)}+C_{B} \sum_{\mathbf{l}, \mathbf{m} \in \mathbf{Z}^{d}} q_{\mathbf{k}, \mathbf{l}, \mathbf{m}} B_{\mathbf{k}, \mathbf{l}, \mathbf{m}}\left(\chi_{\mathbf{l}}^{(n)}, \chi_{\mathbf{m}}^{(n-1)}\right)+d_{\mathbf{k}} \gamma_{\mathbf{k}}\right],
\end{aligned}
$$

with initial condition $\chi_{\mathbf{k}}^{(n)}(0)=\chi_{\mathbf{k}}(0)$ for all $\mathbf{k} \in \mathbf{Z}^{d}$ and $n \in \mathbf{N}$.

Proof. The local existence and uniqueness of solutions for the approximation scheme, follows from the same methods as in the proof of Theorem 3.1, plus an inductive argument in $n \geqslant 0$. The fact that solutions are globally defined follows, again by induction, from the simple estimate

$$
\left|\chi_{\mathbf{k}}^{(n)}(t)\right| \leqslant\|\chi(0)\|_{\infty}+\sup _{t \in[0, T]}\|\gamma\|_{\infty}+\lambda_{\mathbf{k}} \int_{0}^{t} e^{-\lambda_{\mathbf{k}}(t-s)}\left(C_{R}+C_{B}\left\|\chi^{(n-1)}\right\|_{\infty}\right)\left\|\chi^{(n)}\right\|_{\infty} \mathrm{d} s,
$$

which, using induction and Gronwall's lemma, easily gives boundedness of $\left\|\chi^{(n)}\right\|_{\infty}$ in each interval [0,T].

In order to prove that the stochastic representation (5.2) is well defined, we use a comparison argument, as in Section 4 . The comparison equation for the approximation scheme is given by

$$
\dot{\tilde{\chi}}_{\mathbf{k}}^{(n)}=\lambda_{\mathbf{k}}\left[-\tilde{\chi}_{\mathbf{k}}^{(n)}+p_{\mathbf{k}} C_{R} \tilde{\chi}_{\mathbf{k}}^{(n)}+C_{B} \sum_{\mathbf{l}, \mathbf{m} \in \mathbf{Z}^{d}} q_{\mathbf{k}, \mathbf{l}, \mathbf{m}} \tilde{\chi}_{\mathbf{l}}^{(n)} \tilde{\chi}_{\mathbf{m}}^{(n-1)}+d_{\mathbf{k}}\left|\gamma_{\mathbf{k}}\right|\right]
$$

and the evaluation $\mathrm{E}_{\mathbf{k}}\left|R_{n, t}\right|$ is finite as long as the $\tilde{\chi}_{\mathbf{k}}$ are finite. But this follows by the same arguments as in first part of this proof. Again $\mathrm{E}_{\mathbf{k}}\left|R_{n, t}\right| \leqslant \tilde{\chi}_{\mathbf{k}} \leqslant C$ for all $\mathbf{k} \in \mathbf{Z}^{d}$ and all $t \in[0, T]$ with constant $C$ depending only on $T, \chi(0)$, and $\gamma$. 
Finally, the expectations $\mathrm{E}_{\mathrm{k}}\left[R_{n, t}\right]$ do form the unique solution to the approximation scheme by conditioning on the first branch of the tree as in Theorem 3.5.

In the integrable case, that is where $\mathrm{E}_{\mathbf{k}}\left|R_{t}\right|<\infty$, we have immediately from (5.1) that

$$
\lim _{n \rightarrow \infty} \mathrm{E}_{\mathbf{k}}\left[R_{n, t}\right]=\mathrm{E}_{\mathbf{k}}\left[R_{t}\right] .
$$

In particular, when the expectations $\mathrm{E}_{\mathbf{k}}\left[R_{t}\right]$ are bounded over $t \in[0, T]$ and $\mathbf{k} \in \mathbf{Z}^{d}$ this implies the solutions of the approximation scheme converge to those of the original system (2.1). Our interest, however, is in the non-integrable case and we aim to show that convergence of the approximation scheme directly and deduce that the $\operatorname{limit}_{\lim } \rightarrow \infty \mathrm{E}_{\mathbf{k}}\left[R_{n, t}\right]$ exists and defines a stochastic representation for all times $t>0$.

\subsection{Global convergence of the stochastic approximation}

The aim of this section is to give a few details of one example where the approximation scheme defined by the pruned representation converges, even when the direct stochastic representation fails to be integrable. In contrast to the previous section, we use PDE methods. The convergence depends crucially on the equation and how the pruning is done, as not all approximation schemes will converge globally.

For simplicity we work with the one-dimensional Burgers equation with forcing (2.5). In Section 2.2 we recast the equation into our abstract form by considering the weighted Fourier coefficients

$$
\chi_{\mathbf{k}}(t)=w_{\mathbf{k}} u_{\mathbf{k}}(t),
$$

where, as in Section 2.2, the weights are given by $w_{\mathbf{k}}=\left(1 \vee|\mathbf{k}|^{\gamma}\right)$ for some $\gamma>1$. If we assume the Fourier coefficients of the initial condition satisfy

$$
\sup _{\mathbf{k}}\left\{\left|u_{\mathbf{k}}(0)\right| w_{\mathbf{k}}\right\}<\infty
$$

and the forcing function $f$ satisfies

$$
\sup _{\mathbf{k}} \sup _{t \in[0, T]}\left\{\left|f_{\mathbf{k}}(t)\right| w_{\mathbf{k}}\right\}<\infty, \quad \text { for all } T>0,
$$

then Proposition 5.1 implies there is a unique global solution $\chi_{\mathbf{k}}^{(n)}(t)$, given by (5.2), to the approximation equations (5.3).

Theorem 5.2. Assume, in addition to (5.4) and (5.5), that $u(0) \in H^{1}$ and $f \in L_{\mathrm{loc}}^{\infty}\left([0, \infty), L^{\infty}\right)$. Consider the pruned approximation of the previous section. Then the limit

$$
\chi_{\mathbf{k}}(t)=\lim _{n \rightarrow \infty} \chi_{\mathbf{k}}^{(n)}(t)=\lim _{n \rightarrow \infty} \mathrm{E}_{\mathbf{k}}\left[R_{n, t}\right]
$$

exists for all $t \geqslant 0$ and all $\mathbf{k} \in \mathbf{Z}$ and defines a global solution of the Fourier-transformed Burgers equation.

Proof. Define

$$
u_{\mathbf{k}}^{(n)}(t)=w_{\mathbf{k}}^{-1} \chi_{\mathbf{k}}^{(n)}(t) .
$$

Since $\chi^{(n)}$ is bounded we may reconstruct from these coefficients the function

$$
u^{(n)}(t)=\sum_{\mathbf{k} \in \mathbf{Z}} u_{\mathbf{k}}^{(n)}(t) \mathrm{e}^{\mathrm{i} k x} .
$$

Using the representation of Proposition 5.1, we see that, on the level of PDEs, $u^{(n)}$ solves the approximation scheme given by

$$
\left\{\begin{array}{l}
\partial_{t} u^{(0)}=\partial_{x}^{2} u^{(0)} \\
\partial_{t} u^{(n)}=\partial_{x}^{2} u^{(n)}+\partial_{x} u^{(n)} u^{(n-1)}+f \\
u^{(n)}(0)=u(0)
\end{array}\right.
$$


Fix $T>0$ and set $C(f, T)=\sup _{t \in[0, T]}\|f(t)\|_{L^{\infty}}$. We first use a maximum principle argument to show

$$
\sup _{t \in[0, T]}\left\|u^{(n)}(t)\right\|_{L^{\infty}} \leqslant\|u(0)\|_{L^{\infty}}+C(f, T) T \quad \text { for all } n \in \mathbf{N} .
$$

We now derive an a-priori estimate for the solution. The following calculation applies to sufficiently smooth functions and standard approximation techniques imply that the resulting bound holds for the solutions above. Using (5.7), we find

$$
\begin{aligned}
\frac{1}{2} \frac{\mathrm{d}}{\mathrm{d} t}\left\|\partial_{x} u^{(n)}\right\|_{L^{2}}^{2} & =-\left\|\partial_{x}^{2} u^{(n)}\right\|_{L^{2}}^{2}-\int_{0}^{2 \pi} \partial_{x} u^{(n)} u^{(n-1)} \partial_{x}^{2} u^{(n)} \mathrm{d} x-\int_{0}^{2 \pi} \partial_{x}^{2} u^{(n)} f \mathrm{~d} x \\
& \leqslant-\left\|\partial_{x}^{2} u^{(n)}\right\|_{L^{2}}^{2}+C\left\|\partial_{x}^{2} u^{(n)}\right\|_{L^{2}}^{3 / 2}+C\left\|\partial_{x}^{2} u^{(n)}\right\|_{L^{2}},
\end{aligned}
$$

where we have used the Poincaré and Cauchy-Schwartz inequalities. Note that the constant $C>0$ depends only on $T, C(f, T)$, and $u(0)$. Thus we find another constant, also denoted $C$, such that for all $n \in \mathbf{N}$

$$
\sup _{t \in[0, T]}\left\|u^{(n)}(t)\right\|_{H^{1}}^{2} \leqslant C, \quad \int_{0}^{T}\left\|u^{(n)}(t)\right\|_{H^{2}}^{2} \mathrm{~d} t \leqslant C \quad \text { and } \quad \int_{0}^{T}\left\|\partial_{t} u^{(n)}(t)\right\|_{L^{2}}^{2} \mathrm{~d} t \leqslant C .
$$

We now use standard methods to show that we have a solution of the limiting equation (see for example Temam [19]). Indeed by compactness results, there is a subsequence $\left(n_{k}\right)_{k \in \mathbf{N}}$, such that $u^{n_{k}} \rightarrow u$ weakly in $L^{2}\left([0, T], H^{2}\right)$ and $H^{1}\left([0, T], L^{2}\right)$, and strongly in $L^{p}\left([0, T], L^{2}\right)$ for any $p>1$. Thus $u$ is the weak solution of Burgers equation, i.e. it solves the PDE in $L^{2}\left([0, T], L^{2}\right)$. As weak solutions of the Burgers equation are unique, we can neglect the subsequence, since any limiting point of $u^{(n)}$ defines the same solution $u$. Finally, the convergence is strong enough, in order to have all Fourier coefficients convergent. Thus for all $\mathbf{k} \in \mathbf{Z}$ the Fourier coefficients $u_{\mathbf{k}}$ of $u$ are given by

$$
u_{\mathbf{k}}(t)=\lim _{n \rightarrow \infty} u_{\mathbf{k}}^{(n)}(t)=\lim _{n \rightarrow \infty} w_{\mathbf{k}}^{-1} \chi_{\mathbf{k}}^{(n)}(t) .
$$

Remark 5.3. We point out that the assumptions of the previous theorem are by no means optimal. We have used a simplified method of proof, in order to provide an example in a simple context. In particular the constraint on the initial condition can be relaxed. Furthermore, using regularisation properties of the PDE, we can always get sufficiently smooth initial conditions, if we wait a small amount of time.

\section{Acknowledgements}

We wish to thank F. Morandin for some useful conversations on the subject, the anonymous referee for helping us to improve the paper and for letting us know references [6] and [14].

The first and second author wish to thank the Maths Research Centre at the University of Warwick, where part of this work has been prepared, for its warm hospitality.

\section{References}

[1] K.B. Athreya, P.E. Ney, Branching Processes, Die Grundlehren der Mathematischen Wissenschaften, Band 196, Springer-Verlag, New YorkHeidelberg, 1972.

[2] S. Athreya, R. Tribe, Uniqueness for a class of one-dimensional stochastic PDEs using moment duality, Ann. Probab. 28 (4) (2000) 17111734.

[3] Y. Bakhtin, Existence and uniqueness of stationary solutions for 3D Navier-Stokes system with small random forcing via stochastic cascades, J. Statist. Phys. 122 (2) (2006) 351-360.

[4] R. Bhattacharya, L. Chen, S. Dobson, R. Guenther, Ch. Orum, M. Ossiander, E. Thomann, E. Waymire, Majorizing kernels and stochastic cascades with applications to incompressible Navier-Stokes equations, Trans. Amer. Math. Soc. 355 (2003) 5003-5040.

[5] M. Bjørhus, A.M. Stuart, Waveform relaxation as a dynamical system, Math. Comput. 66 (219) (1997) 1101-1117.

[6] L. Chen, S. Dobson, R. Guenther, Ch. Orum, M. Ossiander, E. Thomann, E. Waymire, On Itô's complex measure condition, in: Probability, Statistics and their Applications: Papers in Honor of Rabi Bhattacharya, in: IMS Lecture Notes, vol. 41, 2003, pp. 65-80.

[7] D. Blömker, C. Gugg, M. Raible, Thin-film-growth models: Roughness and correlation functions, Eur. J. Appl. Math. 13 (4) (2002) $385-402$. 
[8] T.E. Harris, The Theory of Branching Processes, Die Grundlehren der Mathematischen Wissenschaften, Band 119, Springer-Verlag, Berlin, 1963.

[9] N. Ikeda, M. Nagasawa, S. Watanabe, Branching Markov processes. I, J. Math. Kyoto Univ. 8 (1968) 233-278.

[10] Y. Le Jan, A.S. Sznitman, Stochastic cascades and 3-dimensional Navier-Stokes equations, Probab. Theory Related Fields 109 (3) (1997) 343-366.

[11] Y. Le Jan, A.S. Sznitman, Cascades aléatoires et équations de Navier-Stokes, C. R. Acad. Sci. Paris Sér. I Math. 324 (7) (1997) 823-826.

[12] J.A. López-Mimbela, A. Wakolbinger, Length of Galton-Watson trees and blow-up of semilinear systems, J. Appl. Probab. 35 (4) (1998) 802-811.

[13] H.P. McKean, Application of Brownian motion to the equation of Kolmogorov-Petrovskii-Piskunov, Comm. Pure Appl. Math. 28 (3) (1975) 323-331.

[14] S. Montgomery-Smith, Finite time blow-up for a Navier-Stokes like equation, Proc. Amer. Math. Soc. 129 (10) (2001) $3025-3029$.

[15] F. Morandin, A resummed branching process representation for a class of nonlinear ODEs, Electron. Comm. Probab. 10 (2005) 1-6.

[16] M. Ossiander, A probabilistic representation of the incompressible Navier-Stokes equation in $\mathbf{R}^{3}$, Probab. Theory Relat. Fields 133 (2) (2005) 267-298.

[17] M. Raible, S.G. Mayr, S.J. Linz, M. Moske, P. Hänggi, K. Samwer, Amorphous thin film growth: Theory compared with experiment, Europhys. Lett. 50 (2000) 61-67.

[18] A.V. Skorokhod, Branching diffusion processes, Theor. Probab. Appl. 9 (1964) 445-449.

[19] R. Temam, Infinite-Dimensional Dynamical Systems in Mechanics and Physics, Applied Mathematical Sciences, vol. 68, Springer-Verlag, New York, 1988

[20] R. Temam, Navier-Stokes Equations and Nonlinear Functional Analysis, CBMS-NSF Regional Conference Series in Applied Mathematics, vol. 41, Society for Industrial and Applied Mathematics (SIAM), Philadelphia, PA, 1983.

[21] E. Waymire, Probability and incompressible Navier-Stokes equations: An overview of some recent developments, Probab. Surveys 2 (2005) $1-32$. 